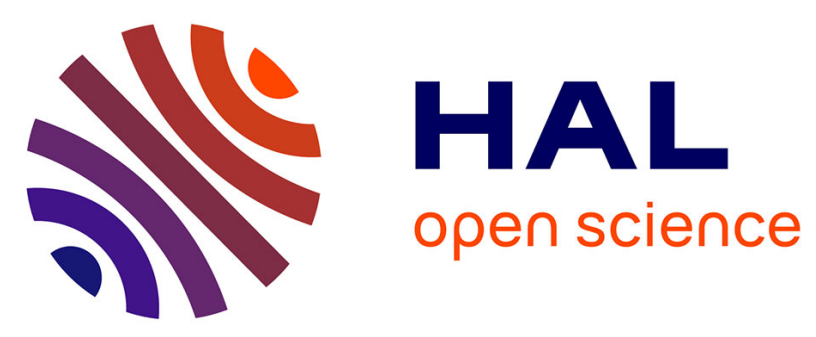

\title{
Dietary omega-3 PUFA improved tubular function after ischemia induced acute kidney injury in mice but did not attenuate impairment of renal function
}

Katharina Rund, Shu Peng, Robert Greite, Cornelius Claassen, Fabian Nolte, Camille Oger, Jean-Marie Galano, Laurence Balas, Thierry Durand, Rongjun Chen, et al.

\section{To cite this version:}

Katharina Rund, Shu Peng, Robert Greite, Cornelius Claassen, Fabian Nolte, et al.. Dietary omega3 PUFA improved tubular function after ischemia induced acute kidney injury in mice but did not attenuate impairment of renal function. Prostaglandins and Other Lipid Mediators, 2019, pp.106386. 10.1016/j.prostaglandins.2019.106386 . hal-02396035v1

\section{HAL Id: hal-02396035 \\ https://hal.science/hal-02396035v1}

Submitted on 5 Dec 2019 (v1), last revised 19 Nov 2021 (v2)

HAL is a multi-disciplinary open access archive for the deposit and dissemination of scientific research documents, whether they are published or not. The documents may come from teaching and research institutions in France or abroad, or from public or private research centers.
L'archive ouverte pluridisciplinaire HAL, est destinée au dépôt et à la diffusion de documents scientifiques de niveau recherche, publiés ou non, émanant des établissements d'enseignement et de recherche français ou étrangers, des laboratoires publics ou privés. 


\section{Journal Pre-proof}

Dietary omega-3 PUFA improved tubular function after ischemia induced acute kidney injury in mice but did not attenuate impairment of renal function

Katharina Rund, Shu Peng, Robert Greite, Cornelius Claaßen, Fabian Nolte, Camille Oger, Jean-Marie Galano, Laurence Balas, Thierry Durand, Rongjun Chen, Faikah Gueler, Nils Helge Schebb

PII: $\quad$ S1098-8823(19)30137-6

DOI: $\quad$ https://doi.org/10.1016/j.prostaglandins.2019.106386

Received Date: $\quad 10$ May 2019

Revised Date: $\quad 9$ August 2019

Accepted Date: $\quad 28$ August 2019

Please cite this article as: Rund K, Peng S, Greite R, Claaßen C, Nolte F, Oger C, Galano J-Marie, Balas L, Durand T, Chen R, Gueler F, Schebb NH, Dietary omega-3 PUFA improved tubular function after ischemia induced acute kidney injury in mice but did not attenuate impairment of renal function, Prostaglandins and Other Lipid Mediators (2019), doi: https://doi.org/10.1016/j.prostaglandins.2019.106386 
This is a PDF file of an article that has undergone enhancements after acceptance, such as the addition of a cover page and metadata, and formatting for readability, but it is not yet the definitive version of record. This version will undergo additional copyediting, typesetting and review before it is published in its final form, but we are providing this version to give early visibility of the article. Please note that, during the production process, errors may be discovered which could affect the content, and all legal disclaimers that apply to the journal pertain. 


\section{Dietary omega-3 PUFA improved tubular function after ischemia induced acute kidney injury in mice but did not attenuate impairment of renal function}

Katharina Rund ${ }^{1 \#}$, Shu Peng ${ }^{2,3 \#}$, Robert Greite ${ }^{2}$, Cornelius Claaßen ${ }^{1}$, Fabian Nolte ${ }^{1}$, Camille Oger $^{4}$, Jean-Marie Galano ${ }^{4}$, Laurence Balas ${ }^{4}$, Thierry Durand ${ }^{4}$, Rongjun Chen ${ }^{2}$, Faikah Gueler#, Nils Helge Schebb ${ }^{1 * \#}$

${ }^{1}$ Chair of Food Chemistry, Faculty of Mathematics and Natural Sciences, University of Wuppertal, Wuppertal, Germany

${ }^{2}$ Nephrology, Hannover Medical School, Hannover, Germany

${ }^{3}$ Department of Thoracic surgery, Tongji hospital, Tongji medical college, Huazhong University of Science and Technology

${ }^{4}$ Institut des Biomolécules Max Mousseron (IBMM), UMR 5247 CNRS, Université de Montpellier, ENSCM, France

\# Authors contributed equally

${ }^{*}$ Corresponding author (Tel: +49 202-439-3457; E-mail: nils@schebb-web.de, postal address: Chair of Food Chemistry, Faculty of Mathematics and Natural Sciences, University of Wuppertal, Gaußstr. 20, 42119 Wuppertal)

Highlights

- Maximal modulation of n3-PUFA and their oxylipins by long chain n3-PUFA feeding

- Apparent PD1 signals in LC-MS/MS analysis seem to result from isobaric interference

- Isoprostanes follow the modulation of parent PUFA in response to feeding: Increase of n3-isoprostanes / decrease of n6-isoprostanes

- Dietary n3-PUFA did not improve overall renal function following ischemic reperfusion injury 
- Moderate protection of tubular function by dietary n3-PUFA and its oxylipins

\section{Abstract}

Background: Acute kidney injury (AKI) is an important complication after major surgery and solid organ transplantation. Here, we present a dietary omega-3 polyunsaturated fatty acid (n3-PUFA) supplementation study to investigate whether pre-treatment can reduce ischemia induced AKI in mice.

Methods: Male 12-14 week old C57BL/6J mice received a linoleic acid rich sunflower oil based standard diet containing 10\% fat (STD) or the same diet enriched with n3-PUFA (containing $1 \%$ EPA and $1 \%$ DHA) (STD+n3). After 14 days of feeding bilateral 30 min renal ischemia reperfusion injury (IRI) was conducted to induce AKI and mice were sacrificed at $24 \mathrm{~h}$. Serum creatinine and blood urea nitrogen (BUN) as well as liver enzyme elevation were measured. Kidney damage was analyzed by histology and immunohistochemistry. Furthermore, pro-inflammatory cytokines (IL-6, MCP-1) were determined by qPCR. FA and oxylipin pattern were quantified in blood and kidneys by GC-FID and LC-MS/MS, respectively.

Results: n3-PUFA supplementation prior to renal IRI increased systemic and renal levels of n3-PUFA. Consistently, eicosanoids and other oxylipins derived from n3-PUFA including precursors of specialized pro-resolving mediators were elevated while n6-PUFA derived mediators such as pro-inflammatory prostaglandins were decreased. Feeding of n3-PUFA did not attenuate renal function impairment, morphological renal damage and inflammation characterized by IL-6 and MCP-1 elevation or neutrophil infiltration. However, the tubular transport marker alpha-1 microglobulin (A1M) was significantly higher expressed in proximal tubular epithelial cells of STD+n3 compared to STD fed mice. This indicates a better integrity of proximal tubular epithelial cells and thus significant protection of tubular function. In addition, heme oxygenase-1 (HO-1) which protects tubular function was also up-regulated in the treatment group receiving n3-PUFA supplemented chow. 
Discussion: We showed that n3-PUFA pre-treatment did not affect overall renal function or renal inflammation in a mouse model of moderate ischemia induced $\mathrm{AKI}$, but tubular transport was improved. In conclusion, n3-PUFA food supplementation altered the oxylipin levels significantly but did not protect from renal function deterioration or attenuate ischemia induced renal inflammation.

\section{Introduction}

Acute kidney injury (AKI) is a frequent complication after major cardiac surgery, solid organ transplantation and also after trauma surgery. Onset of $\mathrm{AKI}$ increases morbidity and mortality of the patients and is characterized by an increase in serum creatinine and/or reduction of the glomerular filtration rate (GFR) [1]. In a review of 25,182 trauma patients post-traumatic AKI was as high as $24 \%$ and these patients had a 3.4 -fold higher risk of death compared to non-AKI patients [2]. Also, after myocardial infarction mortality was 3-fold increased in patients with AKI and incidence of major cardiac events (MACE) was with $26 \%$ higher than in non-AKI patients during the three year follow-up [3]. It has been shown that $\mathrm{AKI}$ is not only an acute event but also increases the risk for chronic kidney disease (CKD) [4]. Even if the serum creatinine elevation returns to normal levels shortly after AKI a significant proportion of patients will develop CKD or will even proceed to end stage renal disease with the need of renal replacement therapy [5]

Renal ischemia reperfusion (IRI) injury due to hypotension, major bleeding and hypoxia causes intrarenal vasoconstriction with activation of pro-oxidative mechanisms, release of proinflammatory cytokines and subsequent leukocyte invasion [6-9]. The severity of AKI correlates with the long-term outcome and progression to CKD [5].

Established strategies to prevent or attenuate AKI even in scheduled surgeries such as cardiac valve replacement are lacking. Several studies discussed a beneficial role of omega-3 polyunsaturated fatty acids (n3-PUFA) in the context of kidney diseases [10-19]. For the prevention of cardiovascular diseases the nutritional-status of n3-PUFA is a well-established risk 
factor $[20,21]$. The higher the incorporation of n3-PUFA, determined by the relative content of EPA and DHA in the cells, typically red blood cells [20, 22], the lower the risk for cardiovascular diseases and mortality [23]. It has to be noted that n3-PUFA are essential constituents of the human diet and that the nutritional status and not the intake of n3-PUFA supplements is related to their physiological effects. Thus, if only the intake of n3-PUFA by supplementation is correlated to the risk for cardiovascular diseases the outcome is less clear [24, 25].

In the context of renal diseases effects of n3-PUFA supplementation on the outcome in kidney transplantation are inconsistent [26]. However, in some experimental AKI models the administration of n3-PUFA correlated with an improved kidney function [10-15].

The molecular mechanisms underlying beneficial effects of n3-PUFA include direct actions, e.g. binding to ion channels or transcription factors, e.g. NFKB or PPARY, thereby reducing the expression of pro-inflammatory and activating the expression of anti-inflammatory genes, respectively. As constituents of membranes, e.g. predominantly in phospholipids, n3-PUFA also impact membrane structure and fluidity [27]. However, it is beyond doubt that a relevant part of the physiological effects of n3-PUFA are mediated by their oxygenated products, i.e. eicosanoids and other oxylipins [27-29]. In the so-called arachidonic acid (ARA) cascade PUFA serve as substrates for enzymatic and non-enzymatic conversion resulting in a multitude of lipid mediators from both n6- and n3-PUFA (Fig. 1) [27, 28, 30]. Enzymatic conversion of PUFA in the ARA cascade comprises three major pathways: (I) cyclooxygenases (COX) lead to the formation of prostanoids and thromboxanes, (II) cytochrome P450 monooxygenases (CYP) give rise to epoxyPUFA and terminal hydroxy-PUFA and (III) lipoxygenases lead via hydroperoxy-PUFA to hydroxyPUFA and leukotrienes [31]. Structurally similar to these enzymatically formed products oxidized PUFA also arise during non-enzymatic autoxidation, e.g. isoprostanes, which also have been shown to possess biological activity $[32,33]$. In the kidney, oxylipins have important functions in (patho-)physiology, e.g. regulation of renal blood flow, glomerular filtration rate and tubular transport function [34-37]. As n3-PUFA compete with ARA for conversion dietary n3-PUFA 
supplementation impacts the overall oxylipin profile resulting in a shift from predominantly proinflammatory, e.g. 2-series prostaglandins, towards n3-PUFA derived oxylipins with less potency or even anti-inflammatory properties [27]. Furthermore, in recent years activity of LOX with n3PUFA have been related to the formation of multiple hydroxylated PUFA, e.g. resolvins, maresins and protectins which have been attributed to be actively involved in the resolution of inflammation [38], though several studies fail to detect them in biological samples [39, 40].

Changes in the oxylipin pattern in the context of kidney function in AKI have been sparsely investigated. Therefore, we used a well characterized model of ischemia induced AKI in mice [41, 42] to study the effect of dietary n3-PUFA (EPA and DHA) supplementation - based on a linoleic acid rich western-like diet [43] for 14 days prior to surgery - on the fatty acid composition and oxylipin pattern in blood and tissue in comparison to renal damage and inflammation at $24 \mathrm{~h}$ after injury. 


\section{Materials and methods}

Chemicals

Oxylipin and deuterated oxylipin standards were purchased from Cayman Chemicals (local distributor: Biomol, Hamburg, Germany). Methyl pentacosanoate (FAME C25:0) was obtained from Santa Cruz Biotechnology (Heidelberg, Germany). PD1 and isoprostane standards were included in the method based on in-house synthesis as described [40, 44]. HPLC grade and LCMS grade methanol (MeOH), LC-MS grade acetonitrile (ACN), LC-MS grade isopropanol, LC-MS grade acetic acid and HPLC grade methyl tert-butyl ether (Acros Organics) were purchased from Fisher Scientific (Schwerte, Germany). Sodium hydrogen phosphate and $n$-hexane (HPLC grade) were obtained from Carl Roth (Karlsruhe, Germany). Ammonium acetate (p.a.) was obtained from Merck (Darmstadt, Germany) and potassium hydroxide (85\%) from Gruessing GmbH (Filsum, Germany). Ethyl acetate, acetyl chloride and potassium carbonate (anhydrous) were purchased from Sigma Aldrich (Schnelldorf, Germany).

Feeding experiment and renal ischemia reperfusion injury in mice

C57BL/6 Jam-ztm male mice (12-14 weeks of age) were obtained from the institute of laboratory animal science (Hannover Medical School, Germany). Mice were cared for in accordance with the institution's guidelines for experimental animal welfare and with the guidelines of the American Physiological Society. All experiments were approved by the animal protection committee of the local authorities (Lower Saxony state department for food safety and animal welfare, LAVES; approval 33.19-42502-04-14/1657). Mice were housed under conventional conditions with a 14/10 h light/dark cycle. Mice were divided into two feeding groups receiving either a sunflower oil based standard diet (STD) or the same diet enriched with n3-PUFA containing $1 \%$ eicosapentaenoic acid (EPA) and $1 \%$ docosahexaenoic acid (DHA) as ethyl esters (10\% each in fat; STD+n3) based on a standard experimental diet (ssniff Spezialdiaeten GmbH, Soest, Germany). Both diets contained in total $10 \%$ fat. The fatty acid composition of the diets is shown 
in the supplementary material (SI, Tab. S1). During the whole feeding period every 2-3 days fresh chow was provided and animals had free access to the food and domestic quality drinking water. After 14 days of feeding, renal IRI was initiated in general isoflurane anesthesia $(5 \%$ induction, $2 \%$ maintenance) combined with iv butorphanol as analgetic treatment. $|R|$ was induced by transient bilateral renal pedicle clamping for 30 min using a non-traumatic vascular clamp [45]. Mice were sacrificed $24 \mathrm{~h}$ after reperfusion by deep general anesthesia and total body perfusion with ice cold PBS [45].

$|R|$ experiments were conducted in three sets each with $n=7-8$ animals in the STD and STD+n3 fed group. In one of these experiments additionally a sham group $(n=5)$ receiving STD chow was included which underwent midline laparotomy but without renal pedicle clamping.

Blood was collected at baseline (before starting the feeding) and at $24 \mathrm{~h}$ after reperfusion. An aliquot of $30 \mu \mathrm{L}$ whole blood was diluted with $150 \mu \mathrm{L}$ deionized water for fatty acid analysis and EDTA-plasma was generated by centrifugation $\left(4000 \times \mathrm{g}, 10 \mathrm{~min}, 4^{\circ} \mathrm{C}\right)$ and stored at $-80^{\circ} \mathrm{C}$ until analysis for clinical chemistry and oxylipins. Kidneys were collected at endpoint, dissected and immediately processed as follows: one piece was fixed in paraformaldehyde for histology, one piece was stored in RNAlater for $\mathrm{qPCR}$ and one piece was shock frozen in liquid nitrogen and stored at $-80^{\circ} \mathrm{C}$ for fatty acid and oxylipin analysis. Renal function (serum-creatinine and BUN) and liver enzymes (aspartate transaminase: AST, alanine aminotransferase: ALT) were measured in EDTA-plasma by an Olympus analyzer (AU400) in an automated fashion according to the manufacturer's instruction.

Histology and immunohistochemistry

The middle part of the kidney was fixed in $4 \%$ paraformaldehyde (PFA) overnight and embedded in paraffin. Two $\mu \mathrm{m}$ paraffin sections were cut and PAS (Perjodic Schiff's Acid) stain according to standard diagnostic protocols was done. For determination of AKI scores a semi-quantitative grading system was used: $0=$ focal $\mathrm{AKI}$ with $<5 \%$ of tubuli of the cortex affected, $1=$ mild AKI with 
$5-25 \%$ of tubuli affected, 2 = moderate AKI with $26-50 \%$ of tubuli affected, $3=$ severe AKI with 51 $75 \%$ of the tubuli affected, $4=$ very severe AKI with $>75 \%$ of tubuli affected.

Immunohistochemistry for neutrophil infiltration (Gr-1 antibody, Biorad), the tubular function marker alpha-1 microglobulin (A1M; gift from Magnus Gram, Lund University) and heme oxygenase-1 (HO-1 antibody; Enzo Life Sciences, Switzerland) was done on paraffin sections. Sections were incubated with trypsin for $15 \mathrm{~min}$ at $37^{\circ} \mathrm{C}$ for antigen retrieval. Nonspecific binding sites were blocked with 10\% normal donkey serum (Jackson ImmunoResearch Lab, West Grove, USA) for $30 \mathrm{~min}$ and then primary antibodies (Invitrogen, California, USA) were incubated for $60 \mathrm{~min}$ at room temperature in the dark. Afterwards secondary antibodies (Invitrogen, California, USA) were incubated for additional $60 \mathrm{~min}$ in the dark. Analysis was performed in a blinded manner using a Leica imaging microscope. Gr-1 positive neutrophil infiltration was scored with a 0-4 grading system in 10 different view fields (VF) per section in 200-fold magnification: $0=$ no infiltrates, $1=$ mild infiltrates with $<5$ cells/VF, $2=$ moderate infiltrates with $6-10$ cells $/ \mathrm{VF}, 3=$ severe infiltration with $11-25$ cells/VF, $4=$ very severe infiltration with $>25$ cells/VF. Semiquantitative analysis of the percentage of $\mathrm{A} 1 \mathrm{M}$ or $\mathrm{HO}-1$ positive proximal tubuli in the cortex was done in 10 different VF per section.

Detection of pro-inflammatory cytokines by qPCR

For mRNA work-up one part of the kidneys was fixed in RNAlater immediately. For total RNA extraction the RNeasy mini kit system (Qiagen, Hilden, Germany) was used and RNA was transcribed with Qiagen mini kits. For quantitative PCR (qPCR) $1 \mu \mathrm{g}$ of DNase-treated total RNA was reverse transcribed using Superscript II reverse transcriptase (Invitrogen) and qPCR was performed on a Lightcycler 420 II (Roche Diagnostics, Penzberg, Germany) using FastStart SybrGreen chemistry. Gene-specific primers for MCP-1 (QT00167832) and IL-6 (QT00098875) were used. For normalization HPRT (QT00166768) was used as housekeeping gene. Quantification was carried out using qgene software. 


\section{Extraction and quantification of fatty acids}

The fatty acid composition was analyzed in diluted whole blood ( $15 \mu \mathrm{L}$ blood $+75 \mu \mathrm{L}$ water) and kidney tissue (15 $\pm 2 \mathrm{mg}$ ) as fatty acid methyl esters using gas chromatography with flame ionization detection (GC-FID) as described with slight modifications [46]. Briefly, $10 \mu \mathrm{L}$ internal standard (FAME C25:0, $750 \mu \mathrm{M}$ ) was added to diluted whole blood and kidney tissue samples. Kidney tissue was homogenized in $300 \mu \mathrm{L} \mathrm{MeOH}$ and $50 \mu \mathrm{L}$ water using two stainless steel beads ( $3 \mathrm{~mm}, 5 \mathrm{~min}, 25 \mathrm{~Hz}$ ) with a vibration ball mill (MM 400, Retsch, Haan, Germany). Lipids were extracted with $\mathrm{MeOH} /$ methyl tert-butyl ether and the lipid extract was trans-esterified to fatty acid methyl esters using methanolic hydrogen chloride. The calculation of the absolute fatty acid concentrations and relative pattern was based on response factors as described [46]. The LLOQ was $0.5 \mu \mathrm{g} / \mathrm{ml}$ for all FAME in the injected solution and the variability in quality control plasma was $<5 \%$ within batch.

Extraction and quantification of oxylipins

Oxylipins in plasma and kidney tissue were extracted using anion exchange Bond Elut Certify II SPE cartridges (Agilent, Waldbronn, Germany) as described with modifications [40, 44]. In the first step of oxylipin extraction $10 \mu \mathrm{L}$ antioxidant solution and internal standards were added to each sample.

For the determination of free oxylipins in plasma (150-200 $\mu \mathrm{L})$ the 2.8 -fold volume of $\mathrm{MeOH}$ was added and the samples were stored at $-80^{\circ} \mathrm{C}$ for $30 \mathrm{~min}$. After centrifugation the supernatant was diluted with disodium hydrogen phosphate buffer yielding a $\mathrm{MeOH}$ content $\leq 18 \%(\mathrm{pH} 6.0)$ and loaded on the preconditioned SPE cartridge.

For analysis of free oxylipins in kidney tissue $(50 \pm 5 \mathrm{mg})$ samples were homogenized with $300 \mu \mathrm{L}$ $\mathrm{MeOH}$ as described [47]. After centrifugation the supernatant was diluted with $2.7 \mathrm{~mL}$ sodium phosphate buffer ( $\mathrm{pH} \mathrm{6.0)} \mathrm{and} \mathrm{loaded} \mathrm{on} \mathrm{the} \mathrm{preconditioned} \mathrm{SPE} \mathrm{cartridge.}$ 
For quantification of total, i.e. free and esterified oxylipins in kidney tissue (20 $\pm 2 \mathrm{mg})$ samples were homogenized in $400 \mu \mathrm{L}$ isopropanol and stored for $30 \mathrm{~min}$ at $-80^{\circ} \mathrm{C}$. Samples were centrifuged and the supernatant was hydrolyzed $(300 \mu \mathrm{L} 1.5 \mathrm{M} \mathrm{KOH}(75 / 25, \mathrm{MeOH} /$ water, $v / v)$, immediately neutralized with acetic acid, diluted with $2 \mathrm{~mL}$ sodium phosphate buffer (pH 5.5) and loaded on the preconditioned SPE cartridge.

The SPE procedure was carried out as described and samples were analyzed by LC-MS/MS (QTRAP, Sciex, Darmstadt, Germany) in scheduled selected reaction monitoring mode following negative electrospray ionization as described $[40,44]$. The lower limit of quantification $[40,44]$ and the analytical variability [48] of the quantified oxylipins are summarized in the SI (Tab. S2).

\section{Data analysis}

Data evaluation and statistical analyses were performed using GraphPad Prism software for Windows (version 5.0, La Jolla California USA). Data are presented as mean \pm standard error of mean (SEM). For oxylipin and fatty acid analysis for the calculation of the mean $1 / 2$ lower limit of quantification (LLOQ) was used if the concentration was below the LLOQ. The concentration was set to LLOQ if the analyte could not be quantified in more than $50 \%$ of the samples in one group. For comparison of two groups two-tailed unpaired student's t-test was used. For multiple comparisons ANOVA with post hoc Tukey correction was applied. Differences were considered significant at a $p$-value $<0.05\left({ }^{*} p<0.05,{ }^{* *} p<0.01,{ }^{* * *} p<0.001\right)$. 


\section{Results}

Bodyweight and food intake of both feeding groups were not different between the STD and the STD+n3 diet.

\section{Blood and tissue pattern of fatty acids and their oxidative metabolites}

After 14 days of feeding an n3-PUFA enriched sunflower oil based diet (1\% EPA and $1 \%$ DHA in the chow, STD+n3) absolute and relative levels of n3-PUFA in the kidney and in the circulation were significantly increased predominantly at the expense of n6-PUFA compared to mice on the STD diet (Fig. $2 \mathrm{~A}+\mathrm{C})$. Considering the very low levels of EPA in tissue and in the circulation of mice on the STD diet, especially relative levels of EPA were massively increased in response to the STD+n3 diet. Interestingly, in whole blood the STD+n3 diet led to an overall decrease in the absolute FA level. The relative concentration of the sum of EPA+DHA, reflecting the endogenous n3-PUFA status, increased 2.5- and 3.7-fold in the kidney and whole blood, respectively (Fig. 2 $\mathrm{B}+\mathrm{D})$ indicating successful modulation of the n3-PUFA content by the supplementation.

Consistent to the changes in the PUFA pattern, levels of eicosanoids and other oxylipins derived from ARA, EPA and DHA were massively altered in response to the STD+n3 diet, while concentrations of oxylipins in the plasma and kidney were almost similar in the IRI treated group compared to the sham group both fed with the STD diet (Fig. 3). Comparing levels of individual oxylipins $24 \mathrm{~h}$ after IRI a similar trend was observed for free metabolites in the plasma as well as for free and esterified oxylipins in kidney tissue, though levels of esterified oxylipins were massively higher than the respective free mediators: Overall STD+n3 diet led to a significant decrease of ARA derived metabolites from all major formation pathways while EPA and DHA derived oxylipins were significantly elevated. Especially, regarding levels of free prostanoids in the kidney tissue, levels of ARA derived prostaglandins such as pro-inflammatory $\mathrm{PGE}_{2}$ or the prostanoid $\mathrm{TxB}_{2}$ were elevated following IRI compared to sham, while STD+n3 feeding caused their significant decrease and concomitant formation of the less potent EPA derived counterparts, i.e. $\mathrm{PGE}_{3}$ and $\mathrm{TxB}_{3}$ (Fig. $3 \mathrm{~B}$ ). Amongst others in the circulation as well as in the kidney also a 
significant increase of 17-HDHA and 18-HEPE, precursors for the formation of pro-resolving mediators (SPM), was observed in response to STD+n3. However, except for DHA derived protectin D1 (PD1) and its isomer PDX no signals above the lower limit of quantification (LLOQ), i.e. $0.25-2 \mathrm{nmol} / \mathrm{kg}$ in kidney tissue and 0.07-0.56 $\mathrm{nM}$ in plasma, were detected for all SPM. LC-MS/MS signals for PD1 indicated relevant apparent concentrations in plasma and kidney of STD+n3 fed mice (Fig, 3, SI Fig. S1). However, the ratios between monitored transitions differed significantly between the authentic standard and the biological samples (SI Fig. S1).

Similar to enzymatically formed oxylipins, levels of prostaglandin-like autoxidation products formed esterified in phospholipids were massively altered by n3-PUFA feeding. Total levels of ARA derived $5(R, S)-\mathrm{F}_{2 \mathrm{t}}$-IsoP were decreased, while EPA and DHA derived $5(R, S)-5-\mathrm{F}_{3 \mathrm{t}}$-IsoP and $4(R, S)-4-\mathrm{F}_{4 \mathrm{t}}-$ NeuroP were elevated in response to STD $+\mathrm{n} 3$. Interestingly, levels of these peroxidation products were lower following IRI compared to sham on the same STD diet (SI Fig. S2).

\section{Renal function, renal morphology and inflammation}

Despite successful n3-PUFA supplementation indicated by the changes of PUFA and oxylipin patterns renal function impairment was similar in both groups. IRI caused a 6-fold elevation of serum creatinine (Fig. 4 I). In line with renal function deterioration, renal damage measured by AKI score was similar in both groups (Fig. 4 A, D, G). Furthermore, Gr-1 positive neutrophil infiltration showed moderate to severe leukocyte infiltration in both groups. On mRNA-level the proinflammatory cytokines IL-6 and MCP-1 were elevated in both groups. The STD+n3 group even showed a trend towards higher values compared to the STD group (Fig. 4 C, F).

\section{Tubular function and heme oxygenase-1 expression}

Alpha-1 microglobulin $(\mathrm{A} 1 \mathrm{M})$ is synthetized in the liver, filtered by the glomeruli and reabsorbed by proximal tubuli. In healthy kidneys $\mathrm{A} 1 \mathrm{M}$ is present in vesicles in the cytoplasm of $60-70 \%$ of the 
tubuli. Upon AKI tubuli have a breakdown in energy metabolism and impaired transport function leading to lower cytoplasm concentration of $A 1 M$ [49]. Due to IRI A1M expression decreased but was significantly higher in the STD+n3 group indicating better preservation of the tubular homeostasis and energy metabolism (Fig. 5 A, C, F). Heme oxygenase-1 (HO-1) is a renoprotective enzyme which is upregulated after $\mathrm{AKI}$ and mediates healing [50]. $\mathrm{HO}-1$ expression was significantly higher in STD+n3 fed mice (Fig. 5 B, D, G). Liver enzymes were elevated after $|R|$ due to distant organ injury [51]. STD+n3 treatment caused less elevation of liver enzymes compared to STD (Fig. 5 E). Overall, we observed some protective effects by the STD+n3 diet in the context of renal IRI (Fig. 5).

\section{Discussion}

Renal IRI is a common cause of AKI which is associated with high morbidity and mortality rates [7]. Particularly in the context of solid organ transplantations IRI is inevitable contributing to impaired allograft function [52]. Cardiac surgery in elderly patients with slightly decreased renal function has incidence rates of $\mathrm{AKI}$ of $\sim 30 \%$ [53]. In the context of scheduled surgeries a dietary intervention would be possible, while the patients are waiting for the surgery. Beneficial effects of n3-PUFA supplementation have been reported, however in the context of kidney transplantation the outcome is inconsistent [26].

In the present study we investigated if dietary n3-PUFA supplementation improves renal function impairment in an ischemia induced AKI model following renal IRI in mice [42]. The control group received a chow, reflecting the omega- 6 rich western diet [43] containing $10 \%$ linoleic acid rich sunflower oil (STD). For the investigation of potential beneficial effects of n3-PUFA supplementation on the base of a western diet, this chow was enriched with n3-PUFA (1\% EPA and $1 \%$ DHA; STD+n3) as previously described [22]. In order to ensure a maximal modulation of the tissue fatty acid composition by the dietary PUFA supplementation a pre-feeding period of 14 days was included prior induction of renal IRI. After 14 days of feeding the STD+n3 chow, the relative fatty acid pattern in the kidney, e.g. relative levels of the sum of saturated fatty acids, 
monounsaturated fatty acids, n6-PUFA and n3-PUFA $(38.3 \%, 10.3 \%, 24.6 \%$ and $26.9 \%$ respectively), was comparable to the relative levels observed after 45 days of feeding the same diet to mice $(38.1 \%, 13.0 \%, 25.2 \%$ and $23.9 \%$ respectively) [22] indicating that a steady-state in the modulation of the kidney fatty acid composition by the feeding was reached after 14 days. This is also consistent with results from feeding fish oil to mice where a steady state of the PUFA status in heart and brain tissue was reached between 1 and 2 weeks [54]. Overall, feeding of the STD+n3 diet led to a massive elevation of absolute and relative levels of n3-PUFA (18:4n3, 20:4n3, 20:5n3, 22:5n3, 22:6n3) with simultaneous decrease in n6-PUFA $(18: 3 n 6,20: 2 n 6,20: 3 n 6,20: 4 n 6,22: 4 n 6$, $22: 5 n 6)$ in the circulation and in the kidney. These changes are in line with previous reports showing extensive modulation of PUFA composition in blood compartments and tissues by n3-PUFA feeding $[22,55-59]$ or by endogenous n3-PUFA accumulation in transgenic fat-1 mice $[22,55,60]$. Compared to the STD chow, in response to the STD+n3 feeding the relative content of the main long-chain PUFA ARA, EPA and DHA in the kidney changed from $16.4 \%$ to $8.6 \%(0.5$ fold), $0.04 \%$ to $4.1 \%$ (99 fold) and $10.1 \%$ to $21.0 \%$ ( 2 fold), respectively. Similar alterations in the renal fatty acid composition were also observed in mice and rats fed chow containing 1-3\% each EPA and DHA (in each case in almost equal amount) [22, 56-58]. Relative levels in the kidney in response to STD+n3 are similar to earlier findings for n3-PUFA feeding in rodents ranging for EPA from $1-4.6 \%$ in mice and from $5.2-9.7 \%$ in rats and for DHA from $6.7-24.6 \%$ in mice and from $5.5-7.8 \%$ in rats depending primarily on the diet composition (e.g. ratio of $n 3 / n 6-P U F A)$. The differences in relative renal levels of EPA and DHA between mice and rats mainly originate from differing PUFA compositions under basal conditions (steady-state without n3-PUFA supplementation) [61]. The relative renal PUFA composition in response to STD+n3 was similar to the relative amount in murine renal phospholipids, especially phosphatidylcholines $(9-12.4,2.3-$ 7.5 and $10-18.1 \%$ of ARA, EPA and DHA respectively) observed after feeding fish oil supplemented chow $[55,59]$, reflecting that phospholipids as main membrane constituents are dominant lipids in the kidney $[62,63]$. 
It should be noted, that the dietary supplementation by STD+n3 led to a more pronounced modulation of the n3-PUFA pattern compared to endogenous synthesis in transgenic fat-1 mice $[22,55]:$ The relative levels in transgenic fat- 1 mice for ARA, EPA and DHA of $20.3 \%, 1 \%$ and $12.1 \%$ in the kidney [22] and of $11.3 \%, 1 \%, 12.9 \%$ in kidney phospholipids, respectively [55] reveal that relative levels reached by STD+n3 feeding are around 4.1 fold and 1.7 fold higher for EPA and $\mathrm{DHA}$ respectively.

The successful modulation of the endogenous n3-PUFA status by the feeding was also clearly indicated by the pronounced changes in \%EPA+DHA levels in blood. In whole blood, which is dominated by the PUFA of erythrocytes, in response to the STD+n3 diet 3.7 fold higher levels of \%EPA+DHA were observed compared to the STD diet $(4.5 \pm 0.1$ in STD vs. $16.9 \pm 0.3 \%$ in STD $+n 3)$. Extrapolating from mice to man, these concentrations reflect a very high endogenous n3-PUFA status, e.g. determined by the omega-3 index, i.e. the relative content of EPA+DHA in membranes of erythrocytes. With a value of $16.9 \%$ the endogenous n3-PUFA levels reached in response to the STD+n3 feeding are significantly higher than those which efficiently reduce overall mortality and the risk for cardiovascular diseases (i.e. an omega-3 index $\geq 8$ ) in men [20, 21]. In contrast, the STD diet reflects the "western-diet" causing a low omega-3 index, even in mice, which more efficiently convert short chain n3-PUFA to long chain n3-PUFA than men [64]. Thus, it can be concluded that the n3-PUFA feeding strategy used in this study reflects the maximal shift in the PUFA pattern which can be reached. On a side note, a well described clinical effect of n3-PUFA is a reduced serum triglyceride concentration $[65,66]$. Indeed, the total FA and thus TG concentration in whole blood was reduced in the animals receiving the STD+n3 compared to the STD diet (Fig. 2).

It is believed that a major portion of beneficial effects of n3-PUFA are mediated by changes in the pattern of oxygenated PUFA, i.e. eicosanoids and other oxylipins [27, 29]. As eicosanoids exert important functions in renal (patho-)physiology including regulation of renal blood flow, glomerular 
filtration rate and tubular transport function [34-37] modulation of the oxylipin pattern by n3-PUFA may also impact kidney function in renal IRI.

Reflecting changes in the PUFA composition, the feeding strategy used in this study resulted in massive alterations of oxylipins in blood and renal tissue from all major formation pathways (COX, LOX, CYP as well as non-enzymatic): ARA derived eicosanoids were significantly decreased while EPA and DHA derived oxylipins were significantly elevated in response to STD+n3 feeding (Fig. 3). Reduction of ARA derived eicosanoids was similar in plasma and kidney (free and total) for all investigated pathways with 40-75\% lower levels after STD+n3 compared to STD feeding. Similarly, DHA derived oxylipins were 1.5-3 fold increased in the kidney, however a more pronounced elevation (7-12 fold) was observed for DHA derived products from 12/15-LOX as well as CYP in plasma. In contrast, EPA derived metabolites were increased to a greater extent (12-53 fold) and in several cases were below the LLOQ in STD while clearly elevated in STD+n3 fed mice. These changes are overall consistent with earlier studies after n3-PUFA (EPA and DHA) supplementation in mice $[13,22,55,67-70]$, rats $[56,67,71-73]$ and humans $[68,74-76]$.

The strongest decrease was observed in kidney levels of ARA derived prostanoids, i.e. PGE 2 (250 vs. $62 \mathrm{nmol} / \mathrm{kg}$ ) and $\mathrm{TxB}_{2}(6.9 \mathrm{vs}<1.2 \mathrm{nmol} / \mathrm{kg})$ and similar to earlier studies with EPA and DHA supplemented chow in mice $[55,67,70]$. Though respective EPA derived counterparts were simultaneously increased resulting levels were considerably lower compared to their corresponding ARA derived prostanoids which is in line with their lower conversion rate by COX in addition to a simple PUFA competition [77]. Considering that EPA derived prostanoids are less bioactive, e.g. $\mathrm{PGE}_{3}$ possesses less affinity towards EP receptors and exhibits lower potency regarding second messenger release [78] and also have been shown to cause less secretion of pro-inflammatory IL-6 [79] compared to $\mathrm{PGE}_{2}$, this clear shift in the renal prostanoid pattern suggests that STD+n3 can contribute to a lower inflammatory status.

Regarding CYP derived metabolites a massive increase in levels of free terminal epoxy-PUFA derived from EPA and DHA, i.e. 17(18)-EpETE and 19(20)-EpDPE, was observed especially in 
plasma, concomitant with a relatively marked decrease (16 vs $5 \mathrm{nmol} / \mathrm{kg}$ in kidney and $1.3 \mathrm{vs}$ $0.4 \mathrm{nM}$ in plasma) in ARA derived 14(15)-EpETrE. Besides displacement of ARA by the n3-PUFA in response to the STD+n3 feeding this profound increase in n3-PUFA derived epoxy-PUFA can be explained by equal or higher conversion rates of DHA and EPA by several CYP isoforms in comparison to ARA $[56,80]$. This marked elevation in n3-PUFA derived terminal epoxy-PUFA in plasma following STD+n3 is similar to their clear increase observed in human plasma after fish oil supplementation [74, 81] supporting their ability to function as indicator for n3-PUFA supplementation as has been suggested [39]. Studies regarding the physiological role of epoxyPUFA in the kidney are mainly focused on ARA derived EpETrE and comprise, e.g. regulation of renal blood flow, inhibition of tubular sodium transport thereby promotion of salt excretion as well as anti-inflammatory effects [82]. Treatment of renal IRI in mice with an inhibitor of the soluble epoxide hydrolase have been shown to ameliorate kidney injury suggesting that epoxy-PUFA have beneficial effects on kidney function in renal IRI [83]. Similarly, n3-PUFA derived EpETE and EpDPE might impact renal physiology, e.g. owing to their higher potency regarding vasodilatory actions compared to EpETrE as well as similar anti-inflammatory effects [82]. Regarding epoxyPUFA, 19(20)-EpDPE reduced kidney fibrosis in a murine unilateral ureteral obstruction (UUO) model [84]. In contrast, in renal IRI administration of 19(20)-EpDPE led to aggravation of kidney damage in mice while 14(15)-EpETrE as well as DHA itself alleviated kidney injury in mice [85]. In the past two decades, a class of multiple hydroxylated PUFA were proposed which actively terminate inflammation, so-called specialized pro-resolving mediators (SPM). From DHA, D-series resolvins as well as protectins and maresins are formed, while EPA gives rise to E-series resolvins [38]. Because of their low biological levels, these mediators are difficult to analyze [40]. Consistent with earlier reports $[22,67,71]$ feeding of the n3-PUFA EPA and DHA led to a massive increase (2-9 fold) in the precursors of SPM, i.e. 17-HDHA, 14-HDHA and 18-HEPE. For example, the 18HEPE content in the kidney in the STD+n3 group was $9.7 \mathrm{nmol} / \mathrm{kg}$ while in the control group it was below the LLOQ (1 nmol/kg). However, except for PD1 all signals for SPM were below the LLOQ, 
i.e. in most cases below $2 \mathrm{nmol} / \mathrm{kg}$ kidney tissue. Thus, it has to be concluded that a 14-day feeding strategy with high n3-PUFA content $(2 \%)$ does not increase the concentration of these mediators above $2 \mathrm{nmol} / \mathrm{kg}$ tissue or $0.56 \mathrm{nM}$ blood. Either the SPM influence physiology in these picomolar, i.e. ppt $(\mathrm{pg} / \mathrm{g})$ concentrations or one has to conclude that they may not play a role in the physiological effects caused by n3-PUFA supplementation.

The signals for free PD1 in the kidney in response to STD+n3 are in a similar range to a previous report detecting PD1 after fish oil supplementation in mice undergoing renal IRI [13]. Analysis of total oxylipins in the kidney suggested even higher levels of PD1 (SI, Fig. S1). As internationally agreed for LC-MS compound identification (e. g. for pesticides [86]), we evaluated two mass transitions, i.e. $m / z 359 \rightarrow 153[13,87-89]$ and $359 \rightarrow 206[87,90,91]$ which also have been previously used for PD1 detection. Particularly in the analysis of the total PD1 concentration in the kidney massive divergence in the area ratio of the two transitions (compared to authentic PD1) results (SI Fig. S1). This clearly suggests that the signals result from isobaric interferences from the sample matrix and not from PD1 (SI Fig. S1). For free oxylipins in the kidney no signal was observed for $\mathrm{m} / \mathrm{z} 359 \rightarrow 206$ and thus, it is even more clear that the signal of PD1 results from interfering matrix and not from the SPM. Isobaric interferences at the retention time of PD1 in reversed-phase HPLC at $m / z 359 \rightarrow 153$ causing an apparent PD1 concentration have been recently also reported for plasma samples after storage [92]. By contrast, the relative intensities of the signals of the PD1-isomer PDX showed identical ratios between authentic standard and samples (SI Fig. S1). Overall our data do not support the earlier described renal formation of PD1 in a mouse model of AKI following feeding a slightly lower dose of n3-PUFA (1.4\% n3-PUFA in chow compared to $2 \%$ in this study) [13].

N3-PUFA are prone to oxidation and may lead to increased oxidative stress. Indeed, in humans receiving a very high dose of DHA $(1.6 \mathrm{~g} / \mathrm{d})$ urinary $15-\mathrm{F}_{2 \mathrm{t}}-\mathrm{Iso}$, a marker of oxidative stress, was increased [93]. This could be even more important in ischemic injury were enhanced oxidative stress plays a key role in pathophysiology [6]. However, our data reveal, that the STD+n3 group 
did not show elevated IsoP levels in the kidney as well as in plasma following IRI compared to the STD diet (Fig. 3, SI Fig. S2). Similarly, lower levels of the autoxidation marker malondialdehyde were observed after renal IRI in the kidney tissue of rats treated with n3-PUFA [11, 12]. However, to comprehensively investigate oxidative stress caused by IRI and its modulation by n3-PUFA supplementation further autoxidation parameters, such as 8-hydroxyguanosine, glutathione, 4 hydroxy-nonenal and expression of (anti)oxidative stress response genes should be monitored in the future.

Altogether feeding of STD+n3 led to a maximal modulation in n3-PUFA and its lipid mediators dominated by n3-PUFA derived oxylipins. Thus, the feeding strategy allows to evaluate the effects of maximal dietary n3-PUFA modulation based on a western diet on acute kidney injury.

Despite the clear effects on oxylipins the STD+n3 diet did not result in improvement of overall renal function or reduction of pro-inflammatory cytokine production or leukocyte infiltration $24 \mathrm{~h}$ after IRI. However, there were measurable effects on protective molecular mechanisms such as up-regulation of $\mathrm{HO}-1$. It has been shown that $\mathrm{HO}-1$ fosters renal regeneration and healing in several models of renal injury [45, 94, 95]. In addition, the tubular transport function which depends on intact energy metabolism of the tubuli was preserved as shown by $\mathrm{A} 1 \mathrm{M}$ staining. There was a direct correlation in previous studies between higher A1M expression and less tissue edema measured by functional MRI [49]. In the current study there was also less ALT elevation which is a marker for impaired liver function. It is well known that AKI causes distant organ injury via enhanced leukocyte infiltration into other organs such as liver, lung, brain, gut [51, 96]. Mechanisms are multifactorial such as release of cytokines and inflammatory mediators, increase in oxidative stress, activation of various immune cells, neutrophil extravasation, generalized endothelial injury, increased vascular permeability and tissue edema formation [97]. In a mouse model of liver IRI also beneficial effects with less tissue damage has been attributed to n3-PUFA treatment [98]. It is conceivable that the moderate beneficial effects would improve patient outcome in longer follow-up by reducing the general inflammatory status. 
In a previous study on renal IRI beneficial effects of dietary n3-PUFA supplementation have been reported [13]. However, the severity of AKI might differ between the studies. In the study by Hassan et al. creatinine elevation was only 2 -3-fold which is less than in our study (6-fold) and might reflect a milder AKI model. In the clinical context interesting and relevant benefits from n3PUFA diets were observed, e.g. they seemed to reduce cardiovascular mortality in patients with chronic kidney disease [99]. In addition, another study showed that n3-PUFA reduced blood pressure and triglyceride levels in patients with CKD stage 3-4 in a placebo-controlled intervention study [100]. Since AKI has a high risk of progression to CKD there might be later beneficial effects on clinical outcome which are missed by the nature of the current study design with a 24 hour follow-up. Further investigations with in-depth analysis of oxylipin levels also at later time points of disease progression might be of interest and shall be addressed in the future.

\section{Conclusion}

In the present study supplementation of a western-style, i.e. linoleic rich diet, with the long chain n3-PUFA EPA and DHA led to a pronounced modulation of the PUFA and oxylipin pattern. However, in a murine model of moderate AKI pre-feeding of n3-PUFA did not attenuate renal function impairment, morphological renal damage and inflammation characterized by proinflammatory cytokine (IL-6 and MCP-1) mRNA up-regulation and neutrophil infiltration. However, also beneficial effects were seen such as preservation of tubular transport function indicated by enhanced alpha-1 microglobulin (A1M) expression in cortical proximal tubular epithelial cells and enhanced up-regulation of tubular heme oxygenase-1 (HO-1) expression which has been correlated to improved renal regeneration [45]. Thus n3-PUFA supplementation might lead to renal protection with respect to long-term damage.

Overall it is concluded that - despite a maximal elevation of endogenous n3-PUFA and n3-PUFA derived oxylipin levels - n3-PUFA supplementation did not result in attenuation of renal function impairment or acute kidney injury within 24 hours in this model. 


\section{Acknowledgement}

We thank Herle Chlebusch and Michaela Beese for excellent technical assistance. Our work was supported by the Fonds der Chemischen Industrie to KR and the German Research Foundation (DFG, Grant SCHE 1801 to NHS and GU 613/1-1 to FG) 
References

[1] Kidney Disease: Improving Global Outcomes (KDIGO) Acute Kidney Injury Work Group, KDIGO Clinical Practice Guideline for Acute Kidney Injury, Kidney International Supplements 2(1) (2012) 1-138.

[2] S. Sovik, M.S. Isachsen, K.M. Nordhuus, C.K. Tveiten, T. Eken, K. Sunde, K.G. Brurberg, S. Beitland, Acute kidney injury in trauma patients admitted to the ICU: a systematic review and metaanalysis, Intensive Care Med (2019).

[3] G. Chalikias, L. Serif, P. Kikas, A. Thomaidis, D. Stakos, D. Makrygiannis, S. Chatzikyriakou, N. Papoulidis, V. Voudris, A. Lantzouraki, M. Muller, S. Arampatzis, S. Konstantinides, D. Tziakas, Long-term impact of acute kidney injury on prognosis in patients with acute myocardial infarction, Int J Cardiol (2019).

[4] C.E. Palant, R.L. Amdur, L.S. Chawla, Long-term consequences of acute kidney injury in the perioperative setting, Curr Opin Anaesthesiol 30(1) (2017) 100-104.

[5] L.S. Chawla, P.W. Eggers, R.A. Star, P.L. Kimmel, Acute Kidney Injury and Chronic Kidney Disease as Interconnected Syndromes, New England Journal of Medicine 371(1) (2014) 58-66.

[6] D.N. Granger, P.R. Kvietys, Reperfusion injury and reactive oxygen species: The evolution of a concept, Redox Biology 6 (2015) 524-551.

[7] J.V. Bonventre, L. Yang, Cellular pathophysiology of ischemic acute kidney injury, The Journal of clinical investigation 121(11) (2011) 4210-4221.

[8] H.K. Eltzschig, T. Eckle, Ischemia and reperfusion-from mechanism to translation, Nature Medicine 17 (2011) 1391.

[9] G.R. Kinsey, L. Li, M.D. Okusa, Inflammation in acute kidney injury, Nephron. Experimental nephrology 109(4) (2008) e102-e107.

[10] D.H. Gwon, T.W. Hwang, J.-Y. Ro, Y.-J. Kang, J.Y. Jeong, D.-K. Kim, K. Lim, D.W. Kim, D.E. Choi, J.-J. Kim, High Endogenous Accumulation of $\omega-3$ Polyunsaturated Fatty Acids Protect against Ischemia-Reperfusion Renal Injury through AMPK-Mediated Autophagy in Fat-1 Mice, International journal of molecular sciences 18(10) (2017) 2081.

[11] M. Ajami, S.H. Davoodi, R. Habibey, N. Namazi, M. Soleimani, H. Pazoki-Toroudi, Effect of DHA+EPA on oxidative stress and apoptosis induced by ischemia-reperfusion in rat kidneys, Fundam Clin Pharmacol 27(6) (2013) 593-602.

[12] S.C. Ashtiyani, H. Najafi, K. Kabirinia, E. Vahedi, L. Jamebozorky, Oral omega-3 fatty acid for reduction of kidney dysfunction induced by reperfusion injury in rats, Iran J Kidney Dis 6(4) (2012) 275-83.

[13] I.R. Hassan, K. Gronert, Acute Changes in Dietary $\omega-3$ and $\omega-6$ Polyunsaturated Fatty Acids Have a Pronounced Impact on Survival following Ischemic Renal Injury and Formation of Renoprotective Docosahexaenoic Acid-Derived Protectin D1, J Immunol 182(5) (2009) 32233232.

[14] M.L. Kielar, D.R. Jeyarajah, X.J. Zhou, C.Y. Lu, Docosahexaenoic acid ameliorates murine ischemic acute renal failure and prevents increases in mRNA abundance for both TNF-alpha and inducible nitric oxide synthase, J Am Soc Nephrol 14(2) (2003) 389-96.

[15] J. Torras, K. Soto, M. Riera, I. Herrero, J. Valles, J.M. Cruzado, J. Alsina, J.M. Grinyo, Changes in renal hemodynamics and physiology after normothermic ischemia in animals supplemented with eicosapentaenoic acid, Transpl Int 9 Suppl 1 (1996) S455-9.

[16] R.G. Fassett, G.C. Gobe, J.M. Peake, J.S. Coombes, Omega-3 Polyunsaturated Fatty Acids in the Treatment of Kidney Disease, American Journal of Kidney Diseases 56(4) (2010) 728-742.

[17] B. Gopinath, D.C. Harris, V.M. Flood, G. Burlutsky, P. Mitchell, Consumption of long-chain n3 PUFA, alpha-linolenic acid and fish is associated with the prevalence of chronic kidney disease, Br J Nutr 105(9) (2011) 1361-8.

[18] X. Huang, B. Lindholm, P. Stenvinkel, J.J. Carrero, Dietary fat modification in patients with chronic kidney disease: n-3 fatty acids and beyond, J Nephrol 26(6) (2013) 960-74. 
[19] J. Hu, Z. Liu, H. Zhang, Omega-3 fatty acid supplementation as an adjunctive therapy in the treatment of chronic kidney disease: a meta-analysis, Clinics (Sao Paulo, Brazil) 72(1) (2017) 5864.

[20] W.S. Harris, C. Von Schacky, The Omega-3 Index: a new risk factor for death from coronary heart disease?, Prev Med 39(1) (2004) 212-20.

[21] C. von Schacky, The Omega-3 Index as a risk factor for cardiovascular diseases, Prostaglandins \& other lipid mediators 96(1) (2011) 94-98.

[22] A.I. Ostermann, P. Waindok, M.J. Schmidt, C.-Y. Chiu, C. Smyl, N. Rohwer, K.-H. Weylandt, N.H. Schebb, Modulation of the endogenous omega-3 fatty acid and oxylipin profile in vivo-A comparison of the fat- 1 transgenic mouse with C57BL/ 6 wildtype mice on an omega-3 fatty acid enriched diet, PloS one 12(9) (2017) e0184470.

[23] W.S. Harris, N.L. Tintle, M.R. Etherton, R.S. Vasan, Erythrocyte long-chain omega-3 fatty acid levels are inversely associated with mortality and with incident cardiovascular disease: The Framingham Heart Study, J Clin Lipidol 12(3) (2018) 718-727 e6.

[24] A.S. Abdelhamid, T.J. Brown, J.S. Brainard, P. Biswas, G.C. Thorpe, H.J. Moore, K.H. Deane, F.K. AlAbdulghafoor, C.D. Summerbell, H.V. Worthington, F. Song, L. Hooper, Omega-3 fatty acids for the primary and secondary prevention of cardiovascular disease, Cochrane Database Syst Rev 11 (2018) CD003177.

[25] T. Aung, J. Halsey, D. Kromhout, H.C. Gerstein, R. Marchioli, L. Tavazzi, J.M. Geleijnse, B. Rauch, A. Ness, P. Galan, E.Y. Chew, J. Bosch, R. Collins, S. Lewington, J. Armitage, R. Clarke, C. for the Omega-3 Treatment Trialists', Associations of Omega-3 Fatty Acid Supplement Use With Cardiovascular Disease Risks: Meta-analysis of 10 Trials Involving 77917 IndividualsMetaanalysis of Associations of Omega-3 Fatty Acids and Cardiovascular RiskMeta-analysis of Associations of Omega-3 Fatty Acids and Cardiovascular Risk, JAMA Cardiology 3(3) (2018) 225233.

[26] A. Tatsioni, M. Chung, Y. Sun, B. Kupelnick, A.H. Lichtenstein, R. Perrone, P. Chew, J. Lau, P.A. Bonis, Effects of fish oil supplementation on kidney transplantation: a systematic review and meta-analysis of randomized, controlled trials, J Am Soc Nephrol 16(8) (2005) 2462-70.

[27] P.C. Calder, Marine omega-3 fatty acids and inflammatory processes: Effects, mechanisms and clinical relevance, Biochimica et Biophysica Acta (BBA) - Molecular and Cell Biology of Lipids 1851(4) (2015) 469-484.

[28] M. Gabbs, S. Leng, J.G. Devassy, M. Monirujjaman, H.M. Aukema, Advances in Our Understanding of Oxylipins Derived from Dietary PUFAs, Advances in Nutrition 6(5) (2015) 513540.

[29] W. Wang, J. Zhu, F. Lyu, D. Panigrahy, K.W. Ferrara, B. Hammock, G. Zhang, w-3 Polyunsaturated fatty acids-derived lipid metabolites on angiogenesis, inflammation and cancer, Prostaglandins \& other lipid mediators 113-115 (2014) 13-20.

[30] C. Gladine, A.I. Ostermann, J.W. Newman, N.H. Schebb, MS-based targeted metabolomics of eicosanoids and other oxylipins: analytical and inter-individual variabilities, Free Radical Biology and Medicine (2019).

[31] M.W. Buczynski, D.S. Dumlao, E.A. Dennis, Thematic Review Series: Proteomics. An integrated omics analysis of eicosanoid biology, Journal of lipid research 50(6) (2009) 1015-1038. [32] J.D. Morrow, K.E. Hill, R.F. Burk, T.M. Nammour, K.F. Badr, L.J. Roberts, 2nd, A series of prostaglandin F2-like compounds are produced in vivo in humans by a non-cyclooxygenase, free radical-catalyzed mechanism, Proceedings of the National Academy of Sciences of the United States of America 87(23) (1990) 9383-7.

[33] J.-M. Galano, Y.Y. Lee, C. Oger, C. Vigor, J. Vercauteren, T. Durand, M. Giera, J.C.-Y. Lee, Isoprostanes, neuroprostanes and phytoprostanes: An overview of 25years of research in chemistry and biology, Progress in lipid research 68 (2017) 83-108.

[34] J.D. Imig, Eicosanoid regulation of the renal vasculature, Am J Physiol Renal Physiol 279(6) (2000) F965-81. 
[35] C.M. Hao, M.D. Breyer, Roles of lipid mediators in kidney injury, Semin Nephrol 27(3) (2007) 338-51.

[36] C.M. Hao, M.D. Breyer, Physiological regulation of prostaglandins in the kidney, Annu Rev Physiol 70 (2008) 357-77.

[37] F. Fan, R.J. Roman, Effect of Cytochrome P450 Metabolites of Arachidonic Acid in Nephrology, J Am Soc Nephrol 28(10) (2017) 2845-2855.

[38] C.N. Serhan, Pro-resolving lipid mediators are leads for resolution physiology, Nature 510 (2014) 92.

[39] R.C. Murphy, Specialized pro-resolving mediators: do they circulate in plasma?, Journal of lipid research 56(9) (2015) 1641-1642.

[40] L. Kutzner, K.M. Rund, A.I. Ostermann, N.M. Hartung, J.-M. Galano, L. Balas, T. Durand, M.S. Balzer, S. David, N.H. Schebb, Development of an Optimized LC-MS Method for the Detection of Specialized Pro-Resolving Mediators in Biological Samples, Frontiers in Pharmacology 10(169) (2019).

[41] K. Hueper, M. Gutberlet, S. Rong, D. Hartung, M. Mengel, X. Lu, H. Haller, F. Wacker, M. Meier, F. Gueler, Acute kidney injury: arterial spin labeling to monitor renal perfusion impairment in mice-comparison with histopathologic results and renal function, Radiology 270(1) (2014) 11724.

[42] F. Gueler, N. Shushakova, M. Mengel, K. Hueper, R. Chen, X. Liu, J.K. Park, H. Haller, G. Wensvoort, S. Rong, A novel therapy to attenuate acute kidney injury and ischemic allograft damage after allogenic kidney transplantation in mice, PloS one 10(1) (2015) e0115709.

[43] T.L. Blasbalg, J.R. Hibbeln, C.E. Ramsden, S.F. Majchrzak, R.R. Rawlings, Changes in consumption of omega-3 and omega- 6 fatty acids in the United States during the 20th century, The American journal of clinical nutrition 93(5) (2011) 950-962.

[44] K.M. Rund, A.I. Ostermann, L. Kutzner, J.-M. Galano, C. Oger, C. Vigor, S. Wecklein, N. Seiwert, T. Durand, N.H. Schebb, Development of an LC-ESI(-)-MS/MS method for the simultaneous quantification of 35 isoprostanes and isofurans derived from the major n3- and n6PUFAs, Analytica chimica acta 1037 (2018) 63-74.

[45] A. Thorenz, K. Derlin, C. Schröder, L. Dressler, V. Vijayan, P. Pradhan, S. Immenschuh, A. Jörns, F. Echtermeyer, C. Herzog, R. Chen, S. Rong, J.H. Bräsen, C. van Kooten, T. Kirsch, C. Klemann, M. Meier, A. Klos, H. Haller, B. Hensen, F. Gueler, Enhanced activation of interleukin10, heme oxygenase-1, and AKT in C5aR2-deficient mice is associated with protection from ischemia reperfusion injury-induced inflammation and fibrosis, Kidney international 94(4) (2018) 741-755.

[46] A.I. Ostermann, M. Müller, I. Willenberg, N.H. Schebb, Determining the fatty acid composition in plasma and tissues as fatty acid methyl esters using gas chromatography - a comparison of different derivatization and extraction procedures, Prostaglandins, Leukotrienes and Essential Fatty Acids 91(6) (2014) 235-241.

[47] H. Gottschall, C. Schmöcker, D. Hartmann, N. Rohwer, K. Rund, L. Kutzner, F. Nolte, A.I. Ostermann, N.H. Schebb, K.H. Weylandt, Aspirin alone and combined with a statin suppresses eicosanoid formation in human colon tissue, Journal of lipid research 59(5) (2018) 864-871.

[48] A.I. Ostermann, T. Greupner, L. Kutzner, Nicole M. Hartung, A. Hahn, J.P. Schuchardt, N.H. Schebb, Intra-individual variance of the human plasma oxylipin pattern: low inter-day variability in fasting blood samples versus high variability during the day, Analytical Methods 10(40) (2018) 4935-4944.

[49] K. Hueper, M. Gutberlet, J.H. Brasen, M.S. Jang, A. Thorenz, R. Chen, B. Hertel, A. Barrmeyer, M. Schmidbauer, M. Meier, S. von Vietinghoff, A. Khalifa, D. Hartung, H. Haller, F. Wacker, S. Rong, F. Gueler, Multiparametric Functional MRI: Non-Invasive Imaging of Inflammation and Edema Formation after Kidney Transplantation in Mice, PloS one 11(9) (2016) e0162705.

[50] F. Gueler, J.K. Park, S. Rong, T. Kirsch, C. Lindschau, W. Zheng, M. Elger, A. Fiebeler, D.

Fliser, F.C. Luft, H. Haller, Statins attenuate ischemia-reperfusion injury by inducing heme 
oxygenase-1 in infiltrating macrophages, The American journal of pathology 170(4) (2007) 11929.

[51] K. Doi, H. Rabb, Impact of acute kidney injury on distant organ function: recent findings and potential therapeutic targets, Kidney international 89(3) (2016) 555-64.

[52] M. Salvadori, G. Rosso, E. Bertoni, Update on ischemia-reperfusion injury in kidney transplantation: Pathogenesis and treatment, World J Transplant 5(2) (2015) 52-67.

[53] W. Jiang, J. Teng, J. Xu, B. Shen, Y. Wang, Y. Fang, Z. Zou, J. Jin, Y. Zhuang, L. Liu, Z. Luo, C. Wang, X. Ding, Dynamic Predictive Scores for Cardiac Surgery-Associated Acute Kidney Injury, J Am Heart Assoc 5(8) (2016).

[54] J.E. Swanson, J. Mark Black, J.E. Kinsella, Dietary menhaden oil: effects on the rate and magnitude of modification of phospholipid fatty acid composition of mouse heart and brain, British Journal of Nutrition 59(3) (1988) 535-545.

[55] D. Kelton, C. Lysecki, H. Aukema, B. Anderson, J.X. Kang, D.W.L. Ma, Endogenous synthesis of $n-3$ PUFA modifies fatty acid composition of kidney phospholipids and eicosanoid levels in the fat-1 mouse, Prostaglandins, Leukotrienes and Essential Fatty Acids 89(4) (2013) 169-177.

[56] C. Arnold, M. Markovic, K. Blossey, G. Wallukat, R. Fischer, R. Dechend, A. Konkel, C. von Schacky, F.C. Luft, D.N. Muller, M. Rothe, W.H. Schunck, Arachidonic acid-metabolizing cytochrome P450 enzymes are targets of \{omega\}-3 fatty acids, The Journal of biological chemistry 285(43) (2010) 32720-33.

[57] G.P. Ossani, V.C. Denninghoff, A.M. Uceda, M.L. Diaz, R. Uicich, A.J. Monserrat, Short-term menhaden oil rich diet changes renal lipid profile in acute kidney injury, Journal of oleo science 64(5) (2015) 497-503.

[58] J.C. Gigliotti, V.A. Benedito, R. Livengood, C. Oldaker, N. Nanda, J.C. Tou, Feeding Different Omega-3 Polyunsaturated Fatty Acid Sources Influences Renal Fatty Acid Composition, Inflammation, and Occurrence of Nephrocalcinosis in Female Sprague-Dawley Rats, Food and Nutrition Sciences Vol.04No.09 (2013) 12.

[59] H.M. Aukema, T. Yamaguchi, H. Takahashi, D.J. Philbrick, B.J. Holub, Effects of dietary fish oil on survival and renal fatty acid composition in murine polycystic kidney disease, Nutrition Research 12(11) (1992) 1383-1392.

[60] J.X. Kang, J. Wang, L. Wu, Z.B. Kang, Transgenic mice: fat-1 mice convert n-6 to n-3 fatty acids, Nature 427(6974) (2004) 504.

[61] P. Couture, A.J. Hulbert, Membrane fatty acid composition of tissues is related to body mass of mammals, The Journal of Membrane Biology 148(1) (1995) 27-39.

[62] R.E. Druilhet, M.L. Overturf, W.M. Kirkendall, Structure of neutral glycerides and phosphoglycerides of human kidney, International Journal of Biochemistry 6(12) (1975) 893-901. [63] G. Rouser, G. Simon, G. Kritchevsky, Species variations in phospholipid class distribution of organs: I. Kidney, liver and spleen, Lipids 4(6) (1969) 599-606.

[64] B.M. Anderson, D.W.L. Ma, Are all n-3 polyunsaturated fatty acids created equal?, Lipids in Health and Disease 8(1) (2009) 33.

[65] W.S. Harris, Omega-3 long-chain PUFA and triglyceride lowering: minimum effective intakes, European Heart Journal Supplements 3(suppl_D) (2001) D59-D61.

[66] T.A.B. Sanders, M.C. Hochland, A comparison of the influence on plasma lipids and platelet function of supplements of $\omega 3$ and $\omega 6$ polyunsaturated fatty acids, British Journal of Nutrition 50(3) (1983) 521-529.

[67] J.G. Devassy, T. Yamaguchi, M. Monirujjaman, M. Gabbs, A. Ravandi, J. Zhou, H.M. Aukema, Distinct effects of dietary flax compared to fish oil, soy protein compared to casein, and sex on the renal oxylipin profile in models of polycystic kidney disease, Prostaglandins, Leukotrienes and Essential Fatty Acids 123 (2017) 1-13.

[68] X. Zhang, N. Yang, D. Ai, Y. Zhu, Systematic metabolomic analysis of eicosanoids after omega-3 polyunsaturated fatty acid supplementation by a highly specific liquid chromatographytandem mass spectrometry-based method, J Proteome Res 14(4) (2015) 1843-53. 
[69] M.G. Balvers, K.C. Verhoeckx, S. Bijlsma, C.M. Rubingh, J. Meijerink, H.M. Wortelboer, R.F. Witkamp, Fish oil and inflammatory status alter the n-3 to n-6 balance of the endocannabinoid and oxylipin metabolomes in mouse plasma and tissues, Metabolomics 8(6) (2012) 1130-1147.

[70] L.S. Gallon, U.O. Barcelli, Measurement of prostaglandin E3 and other eicosanoids in biologic samples using high pressure liquid chromatography and radioimmunoassay, Prostaglandins 31(2) (1986) 217-225.

[71] S. Leng, T. Winter, H.M. Aukema, Dietary ALA, EPA and DHA have distinct effects on oxylipin profiles in female and male rat kidney, liver and serum, The Journal of Nutritional Biochemistry 57 (2018) 228-237.

[72] H.M. Aukema, J. Lu, F. Borthwick, S.D. Proctor, Dietary fish oil reduces glomerular injury and elevated renal hydroxyeicosatetraenoic acid levels in the JCR:LA-cp rat, a model of the metabolic syndrome, Br J Nutr 110(1) (2013) 11-9.

[73] N.H. Ibrahim, Y. Jia, J.G. Devassy, T. Yamaguchi, H.M. Aukema, Renal cyclooxygenase and lipoxygenase products are altered in polycystic kidneys and by dietary soy protein and fish oil treatment in the Han:SPRD-Cy rat, Molecular nutrition \& food research 58(4) (2014) 768-81.

[74] R. Fischer, A. Konkel, H. Mehling, K. Blossey, A. Gapelyuk, N. Wessel, C. von Schacky, R. Dechend, D.N. Muller, M. Rothe, F.C. Luft, K. Weylandt, W.H. Schunck, Dietary omega-3 fatty acids modulate the eicosanoid profile in man primarily via the CYP-epoxygenase pathway, Journal of lipid research 55(6) (2014) 1150-64.

[75] A.M. Zivkovic, J. Yang, K. Georgi, C. Hegedus, M.L. Nording, A. O'Sullivan, J.B. German, R.J. Hogg, R.H. Weiss, C. Bay, B.D. Hammock, Serum oxylipin profiles in IgA nephropathy patients reflect kidney functional alterations, Metabolomics 8(6) (2012) 1102-1113.

[76] N.H. Schebb, A.I. Ostermann, J. Yang, B.D. Hammock, A. Hahn, J.P. Schuchardt, Comparison of the effects of long-chain omega-3 fatty acid supplementation on plasma levels of free and esterified oxylipins, Prostaglandins \& other lipid mediators 113-115 (2014) 21-29.

[77] W.L. Smith, Y. Urade, P.-J. Jakobsson, Enzymes of the Cyclooxygenase Pathways of Prostanoid Biosynthesis, Chemical reviews 111(10) (2011) 5821-5865.

[78] M. Wada, C.J. DeLong, Y.H. Hong, C.J. Rieke, I. Song, R.S. Sidhu, C. Yuan, M. Warnock, A.H. Schmaier, C. Yokoyama, E.M. Smyth, S.J. Wilson, G.A. FitzGerald, R.M. Garavito, D.X. Sui, J.W. Regan, W.L. Smith, Enzymes and Receptors of Prostaglandin Pathways with Arachidonic Acid-derived Versus Eicosapentaenoic Acid-derived Substrates and Products, Journal of Biological Chemistry 282(31) (2007) 22254-22266.

[79] D. Bagga, L. Wang, R. Farias-Eisner, J.A. Glaspy, S.T. Reddy, Differential effects of prostaglandin derived from omega- 6 and omega-3 polyunsaturated fatty acids on COX-2 expression and IL-6 secretion, Proceedings of the National Academy of Sciences of the United States of America 100(4) (2003) 1751-1756.

[80] J.P. Schuchardt, S. Schmidt, G. Kressel, I. Willenberg, B.D. Hammock, A. Hahn, N.H. Schebb, Modulation of blood oxylipin levels by long-chain omega-3 fatty acid supplementation in hyperand normolipidemic men, Prostaglandins, Leukotrienes and Essential Fatty Acids 90(2) (2014) 2737.

[81] C. Skarke, N. Alamuddin, J.A. Lawson, X. Li, J.F. Ferguson, M.P. Reilly, G.A. FitzGerald, Bioactive products formed in humans from fish oils, Journal of lipid research 56(9) (2015) 180820.

[82] C. Westphal, A. Konkel, W.H. Schunck, Cytochrome p450 enzymes in the bioactivation of polyunsaturated Fatty acids and their role in cardiovascular disease, Adv Exp Med Biol 851 (2015) 151-87.

[83] J.P. Lee, S.H. Yang, H.Y. Lee, B. Kim, J.Y. Cho, J.H. Paik, Y.J. Oh, D.K. Kim, C.S. Lim, Y.S. $\mathrm{Kim}$, Soluble epoxide hydrolase activity determines the severity of ischemia-reperfusion injury in kidney, PloS one 7(5) (2012) e37075.

[84] A. Sharma, M.A. Hye Khan, S.P. Levick, K.S. Lee, B.D. Hammock, J.D. Imig, Novel Omega3 Fatty Acid Epoxygenase Metabolite Reduces Kidney Fibrosis, Int J Mol Sci 17(5) (2016). 
[85] B.Q. Deng, Y. Luo, X. Kang, C.B. Li, C. Morisseau, J. Yang, K.S.S. Lee, J. Huang, D.Y. Hu, M.Y. Wu, A. Peng, B.D. Hammock, J.Y. Liu, Epoxide metabolites of arachidonate and docosahexaenoate function conversely in acute kidney injury involved in GSK3beta signaling, Proceedings of the National Academy of Sciences of the United States of America 114(47) (2017) $12608-12613$.

[86] Guidance document on analytical quality control and method validation procedures for pesticide residues and analysis in food and feed., in: E. Commission (Ed.) SANTE/11813/2017, 2017.

[87] A. Barden, E. Mas, K.D. Croft, M. Phillips, T.A. Mori, Short-term n-3 fatty acid supplementation but not aspirin increases plasma proresolving mediators of inflammation, Journal of lipid research 55(11) (2014) 2401-7.

[88] A. Toewe, L. Balas, T. Durand, G. Geisslinger, N. Ferreirós, Simultaneous determination of PUFA-derived pro-resolving metabolites and pathway markers using chiral chromatography and tandem mass spectrometry, Analytica chimica acta 1031 (2018) 185-194.

[89] J.T. English, P.C. Norris, R.R. Hodges, D.A. Dartt, C.N. Serhan, Identification and Profiling of Specialized Pro-Resolving Mediators in Human Tears by Lipid Mediator Metabolomics, Prostaglandins, Leukotrienes and Essential Fatty Acids 117 (2017) 17-27.

[90] K.A. Massey, A. Nicolaou, Lipidomics of oxidized polyunsaturated fatty acids, Free radical biology \& medicine 59 (2013) 45-55.

[91] M. Masoodi, A.A. Mir, N.A. Petasis, C.N. Serhan, A. Nicolaou, Simultaneous lipidomic analysis of three families of bioactive lipid mediators leukotrienes, resolvins, protectins and related hydroxy-fatty acids by liquid chromatography/electrospray ionisation tandem mass spectrometry, Rapid communications in mass spectrometry : RCM 22(2) (2008) 75-83.

[92] H.S. Jonasdottir, H. Brouwers, R.E.M. Toes, A. loan-Facsinay, M. Giera, Effects of anticoagulants and storage conditions on clinical oxylipid levels in human plasma, Biochimica et Biophysica Acta (BBA) - Molecular and Cell Biology of Lipids 1863(12) (2018) 1511-1522.

[93] N. Guillot, E. Caillet, M. Laville, C. Calzada, M. Lagarde, E. Vericel, Increasing intakes of the long-chain omega-3 docosahexaenoic acid: effects on platelet functions and redox status in healthy men, FASEB J 23(9) (2009) 2909-16.

[94] G.M. Goncalves, M.A. Cenedeze, C.Q. Feitoza, P.M. Wang, A.P. Bertocchi, M.J. Damiao, H.S. Pinheiro, V.P. Antunes Teixeira, M.A. dos Reis, A. Pacheco-Silva, N.O. Camara, The role of heme oxygenase 1 in rapamycin-induced renal dysfunction after ischemia and reperfusion injury, Kidney international 70(10) (2006) 1742-9.

[95] J.F. Ndisang, S. Tiwari, Mechanisms by which heme oxygenase rescue renal dysfunction in obesity, Redox Biol 2 (2014) 1029-37.

[96] J.F. Doyle, L.G. Forni, Acute kidney injury: short-term and long-term effects, Crit Care 20(1) (2016) 188.

[97] W. Druml, Systemic consequences of acute kidney injury, Curr Opin Crit Care 20(6) (2014) 613-9.

[98] D.A. Raptis, P. Limani, J.H. Jang, U. Ungethum, C. Tschuor, R. Graf, B. Humar, P.A. Clavien, GPR120 on Kupffer cells mediates hepatoprotective effects of omega3-fatty acids, J Hepatol 60(3) (2014) 625-32.

[99] V.M. Saglimbene, G. Wong, A. van Zwieten, S.C. Palmer, M. Ruospo, P. Natale, K. Campbell, A. Teixeira-Pinto, J.C. Craig, G.F.M. Strippoli, Effects of omega-3 polyunsaturated fatty acid intake in patients with chronic kidney disease: Systematic review and meta-analysis of randomized controlled trials, Clin Nutr (2019).

[100] T.A. Mori, V. Burke, I. Puddey, A. Irish, C.A. Cowpland, L. Beilin, G. Dogra, G.F. Watts, The effects of [omega]3 fatty acids and coenzyme Q10 on blood pressure and heart rate in chronic kidney disease: a randomized controlled trial, J Hypertens 27(9) (2009) 1863-72. 


\section{Figure Captions}

Fig. 1: Simplified overview of the biosynthesis of arachidonic acid (20:4n6, ARA) from linoleic acid (18:2n6, LA) and eicosapenatenoic acid (20:5n3, EPA) and docosahexaenoic acid (20:6n3, DHA) from $\alpha$-linolenic acid (18:3n3, ALA) and selected pathways for their oxygenation within the ARA cascade.

COX - cyclooxygenase, LOX - lipoxygenase, CYP - cytochrome P450 monooxygenase, Tx thromboxane; HETE - hydroxyeicosatetraenoic acid; HEPE - hydroxyeicosapentaenoic acid; HDHA - hydroxydocosahexaenoic acid; EpETrE - epoxyeicosatrienoic acid; EpETE epoxyeicosatetraenoic acid; EpDPE - epoxydocosapentaenoic acid; IsoP - isoprostanes; NeuroP - neuroprostanes; Rv - resolvin; Lx - lipoxin; PD - protectin; MaR - maresin

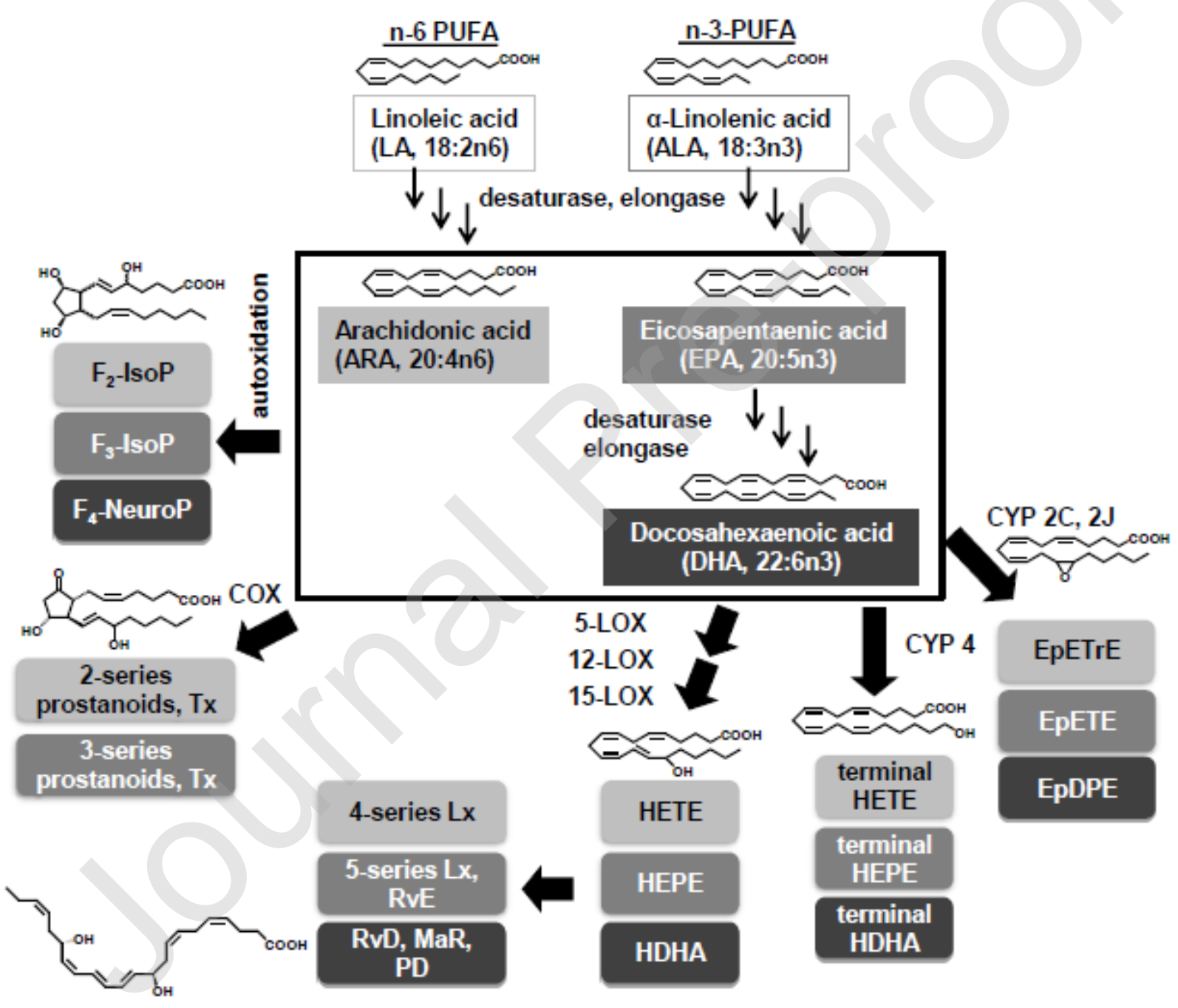


Fig. 2: Fatty acid composition in (A) kidney tissue and (C) whole blood $24 \mathrm{~h}$ after renal IRI in mice was shifted towards higher levels of n3-PUFA, while n6-PUFA decreased after 14 days of feeding a sunflower oil based diet enriched with EPA and DHA (STD+n3) compared to the same diet without EPA and DHA (STD). Consistently relative levels of EPA and DHA of all detected FA in (B) kidney tissue and (D) whole blood were significantly higher in mice on the STD+n3 diet. Shown are mean \pm SEM, $n=14,{ }^{* *} p<0.01,{ }^{* * *} p<0.001$.

(A) kidney tissue

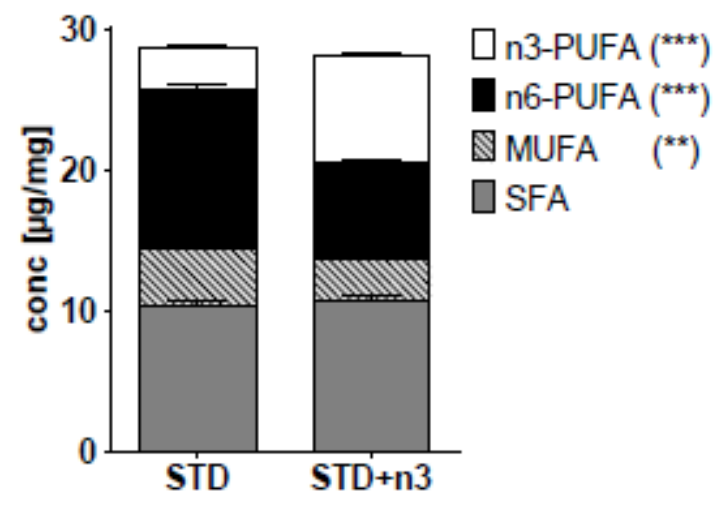

(B) kidney tissue

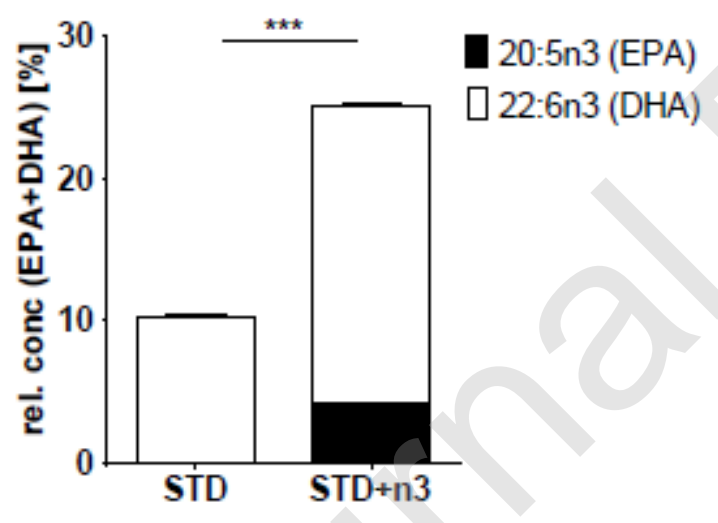

(C) whole blood

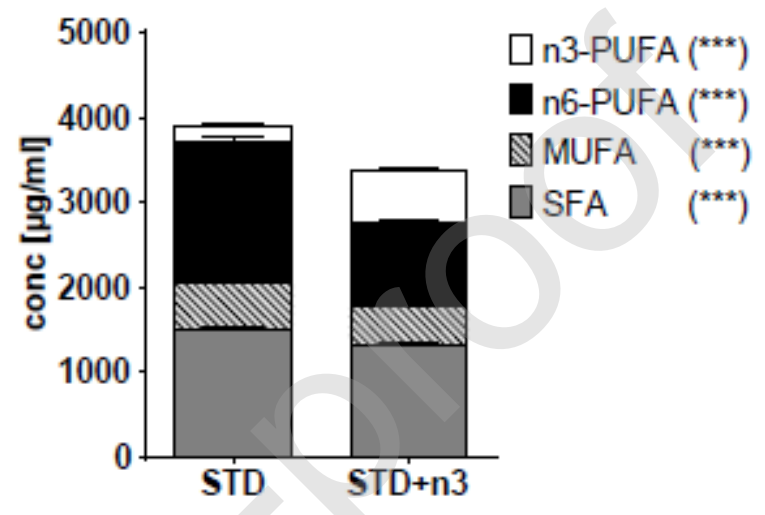

(D) whole blood

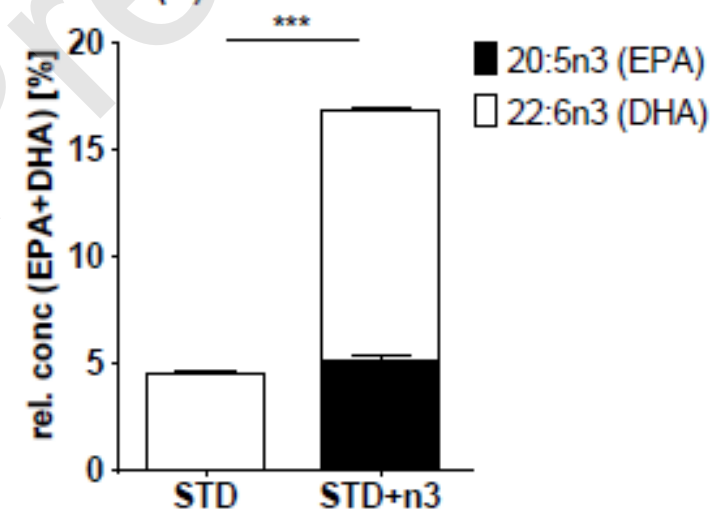

Fig. 3: Concentration of free oxylipins in (A) plasma and (B) kidney tissue as well as (C) total, i.e. free and esterified oxylipins in kidney tissue. Shown are concentrations of selected (iso-) prostanoids, 5-LOX, 12/15-LOX, CYP4 and CYP2 products of ARA, EPA and DHA as well as 18HEPE and PD1. Except for the sham group all patterns are determined in mice $24 \mathrm{~h}$ after renal IRI on a STD or STD+n3 diet. Feeding of the STD+n3 led to an increase of EPA and DHA derived lipid mediators while ARA derived oxylipins were decreased. Shown are mean \pm SEM (sham $n=5$, (A) STD: $n=19$, STD+n3: $n=18$; (B) STD: $n=13$, STD+n3: $n=14$; (C) STD: $n=7$, STD+n3: $n=7$ ). The lower limit of quantification (LLOQ) for those oxylipins is indicated by a dashed line in case it was not exceeded in $>50 \%$ of the samples per group. Statistical differences were determined between STD and STD+n3. 


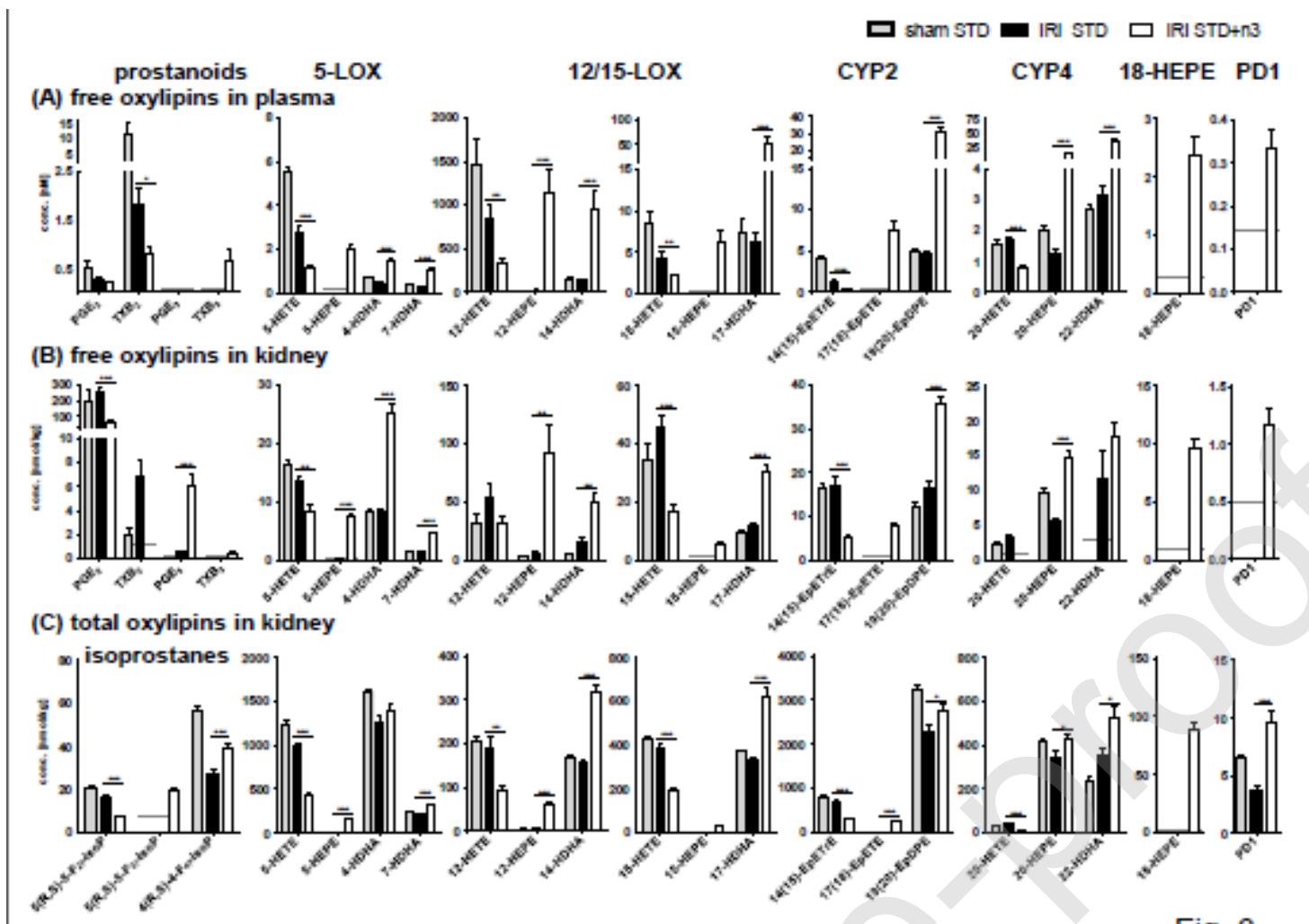

Fig. 3

Fig. 4: Acute kidney injury and inflammation. Renal damage after IRI was comparable in STD and STD+n3 fed mice (A, D, G; STD: $n=18$, STD+n3: $n=21$ ). Neutrophil infiltration to the outer medulla was present in both groups without differences (B, E, H; STD: $n=18$, STD+n3: $n=21$ ). Renal function was significantly impaired after IRI in both groups (I; STD: $n=19$, STD+n3: $n=21$, sham $\mathrm{n}=5$ ). Relative expression of (C) IL-6 and (F) MCP-1 mRNA in kidney tissue was significantly upregulated at $24 \mathrm{~h}$ after $\mathrm{IRI}$ in both groups without differences (STD: $n=7, S T D+n 3: n=7$ ). Shown are mean \pm SEM. 

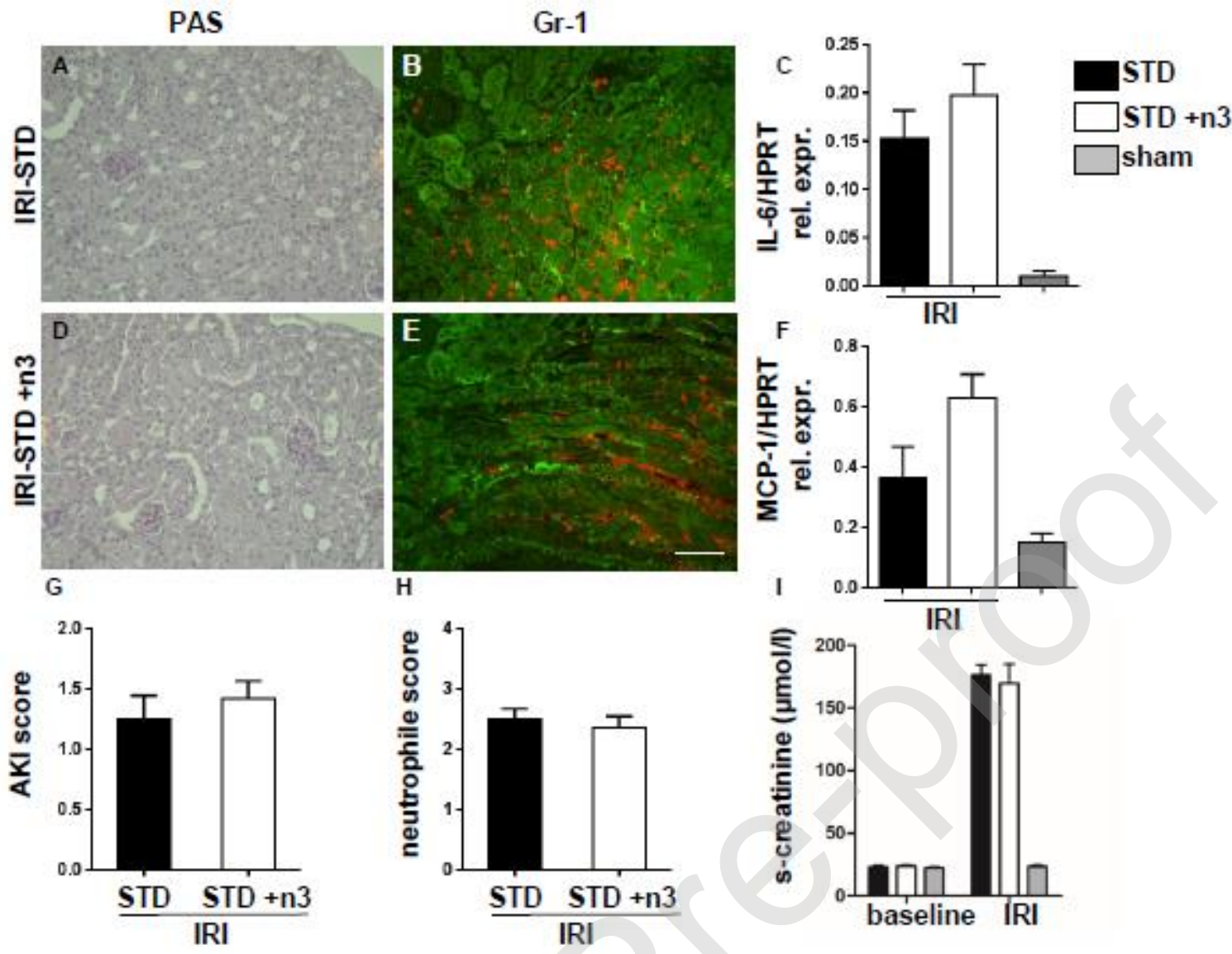

Fig. 5: Tubular transport and tubular regeneration. A1M expression was significantly higher after STD+n3 diet which indicates preserved tubular transport function $(\mathbf{A}, \mathbf{C}, \mathbf{F}$, STD: $\mathrm{n}=18$, STD+n3: $\mathrm{n}=14$ ). In addition, HO-1 expression was significantly upregulated in the STD+n3 fed group (B, $\mathbf{D}$, G; STD: $n=7, S T D+n 3 n=7$ ) which correlates with tubular regeneration. Noteworthy, the elevation of the liver enzyme ALT was attenuated (E) by the STD+n3 diet and could point towards reduced distant organ injury (STD: $n=19$, STD $+n 3 n=21$, sham $n=5$ ). Shown are mean \pm SEM, * $p<0.05$. 

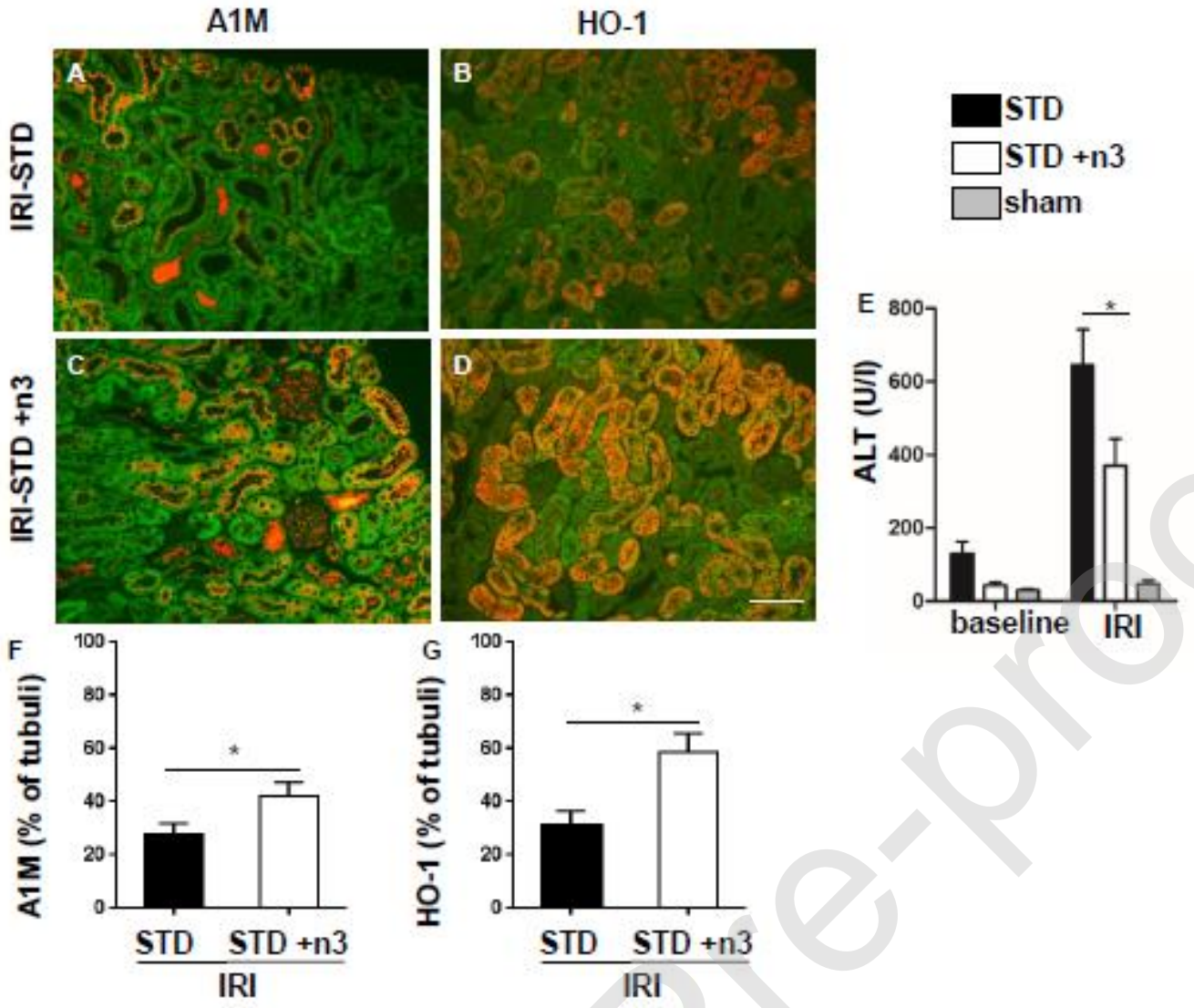